\title{
Trombólisis intraparo en tromboembolia pulmonar masiva
}

\author{
Nicolás Wilches ${ }^{a, *}$, María Claudia Rojas ${ }^{a}$, Ana Milena Mesa ${ }^{a}$, Luis Alfonso Fajardo ${ }^{b}$ \\ y Catalina Gallego ${ }^{b}$
}

a Medicina de Urgencias, Universidad CES, Medellín, Colombia

b Clínica Cardio VID, Medellín, Colombia

Recibido el 29 de marzo de 2017; aceptado el 19 de julio de 2017

Disponible en Internet el 20 de noviembre de 2017

\section{PALABRAS CLAVE \\ Tromboembolia \\ pulmonar; \\ Trombólisis; \\ Paro \\ cardiorrespiratorio}

\section{KEYWORDS}

Pulmonary embolism; Thrombolysis; Cardiopulmonary resuscitation
Resumen La tromboembolia pulmonar forma parte de la enfermedad tromboembólica venosa, junto con la trombosis venosa profunda. Es una patología de alta morbimortalidad, principalmente si no se diagnostica y/o trata de manera oportuna. El diagnóstico es difícil debido a su presentación clínica variable y poco específica.

En el contexto de tromboembolia pulmonar de alto riesgo, que se presenta como una condición que amenaza la vida y requiere intervención inmediata para impactar el pronóstico del paciente, la trombólisis constituye una alternativa en quienes cursen con inestabilidad hemodinámica o incluso parada cardiaca, que no tengan contraindicaciones para su realización. Se presenta el caso de un hombre de 54 años quien consultó al servicio de urgencias tras presentar síncope y posteriormente dolor torácico, precedido por 15 días de dolor y edema en pantorrilla. Sufrió deterioro y evolucionó a parada cardiopulmonar. Se diagnosticó tromboembolia pulmonar de alto riesgo mediante ecocardiograma; se iniciaron maniobras de reanimación y, de manera paralela, se administró trombolítico. Posteriormente, respondió de manera satisfactoria a la terapia.

(c) 2017 Sociedad Colombiana de Cardiología y Cirugía Cardiovascular. Publicado por Elsevier España, S.L.U. Este es un artículo Open Access bajo la licencia CC BY-NC-ND (http:// creativecommons.org/licenses/by-nc-nd/4.0/).

Thrombolysis during cardiopulmonary resuscitation in massive pulmonary embolism

Abstract Pulmonary thromboembolism (PE) is part of venous thromboembolic disease, along with deep venous thrombosis (DVT). It has a high morbidity and mortality, particularly if it is not diagnosed and / or treated in a timely manner. The diagnosis is difficult due to its variable and unspecific clinical presentation.

In the context of high-risk PE, which presents as a life-threatening condition and requires immediate intervention to have an impact on the prognosis of patients, thrombolysis is an

\footnotetext{
* Autor para correspondencia.

Correo electrónico: nicowilches@gmail.com (N. Wilches).
} 
alternative in those with haemodynamic instability or even cardiac arrest, if they have no contraindications for performing this. The case is presented of a 54 year old man who consulted in the emergency department after presenting with syncope followed by chest pain, preceded by 15 days of pain and oedema in the calf. The patient deteriorated rapidly, evolving to cardiopulmonary arrest. A high-risk PE was diagnosed by echocardiography, resuscitation manoeuvres were initiated, and thrombolytic therapy given in parallel. Subsequently the patient had a satisfactory outcome with the therapy.

(c) 2017 Sociedad Colombiana de Cardiología y Cirugía Cardiovascular. Published by Elsevier España, S.L.U. This is an open access article under the CC BY-NC-ND license (http:// creativecommons.org/licenses/by-nc-nd/4.0/).

\section{Introducción}

La enfermedad tromboembólica venosa comprende el espectro de tres entidades básicamente: la tromboembolia pulmonar, la trombosis venosa profunda y el síndrome postflebítico. La tromboembolia pulmonar ocurre cuando un fragmento de trombo viaja libre, proveniente de la circulación venosa de los miembros inferiores; en el $90 \%$ de los casos, pasa a través del lado derecho del corazón y se aloja en las arterias pulmonares. Este trombo puede generar una obstrucción parcial o completa de las arterias pulmonares o alguna de sus ramas (lo cual explica las grandes diferencias en su presentación clínica ${ }^{1}$, que en su expresión más severa produce falla cardiaca, como resultado de un incremento en las presiones del ventrículo derecho, alterando su función y posteriormente deteriorando la función del ventrículo izquierdo. El deterioro de la función cardiaca se presenta en las primeras dos horas del inicio de los síntomas en dos tercios de los casos de tromboembolia pulmonar fatal ${ }^{2}$.

La enfermedad tromboembólica venosa es la tercera causa de enfermedad cardiovascular; su incidencia oscila entre 100-200 por cada 100.000 habitantes en las series europeas y norteamericanas; en el caso de la tromboembolia pulmonar, la incidencia se estima en 95 por cada 100.000 habitantes $^{1,3}$. En 2007, Cohen et al., publicaron datos de seis países europeos, en los que reportaron que hasta el $93 \%$ de las muertes en contexto de enfermedad venoembólica fueron secundarias a tromboembolia pulmonar fatal o trombosis venosa profunda no reconocidas previamente, lo que implica que para este grupo de patologías la falla en el diagnóstico e inicio de tratamiento pertinente, lleva a un aumento en la mortalidad, que puede ser tan importante como de cuatro a seis veces ${ }^{3}$. Se describe en la literatura que entre el 8 y el $13 \%$ de los arrestos cardiacos inexplicados son causados por tromboembolia pulmonar, dato que debe interpretarse de la mano de aquellos de los diferentes registros de tromboembolia pulmonar en los cuales se ha encontrado que entre el 4,2 y el $18 \%$ de pacientes con tromboembolia pulmonar cursan con inestabilidad hemodinámica, de los cuales hasta el $65 \%$ fallece $^{4-6}$.

La tromboembolia pulmonar tiene una amplia variedad de características clínicas que van desde la ausencia de síntomas o síntomas inespecíficos, hasta shock o paro cardiorrespiratorio. El síntoma más común es la disnea (73\%) seguida del dolor pleurítico (66\%) y la tos (37\%) según datos reportados en el estudio PIOPED ${ }^{7}{ }^{7}$. Otros hallazgos menos comunes incluyen arritmias transitorias o persistentes como fibrilación auricular, colapso hemodinámico y síncope, con reportes de menos del $10 \%$ cada uno; sin embargo este último ha sido subvalorado ${ }^{8}$.

Ante un paciente con posible tromboembolia pulmonar, se debe realizar un examen físico minucioso e igualmente investigar factores de riesgo y antecedentes relevantes. La evaluación inicial de probabilidad para tromboembolia pulmonar se hace mediante una de las escalas validadas: Wells, Génova o la versión simplificada de las mismas. Una vez se tenga objetivada la probabilidad de tromboembolia pulmonar según las escalas en mención, se ordenan estudios complementarios como electrocardiograma, Dímero D y angiotomografía de arterias pulmonares ${ }^{9}$.

La trombólisis sistémica disminuye la mortalidad y se convierte en una opción de tratamiento en pacientes con tromboembolia pulmonar de alto riesgo, beneficio que se basa en la restauración rápida del flujo sanguíneo pulmonar y que por ende implica evitar la sobrecarga ventricular derecha y el choque posterior. El estudio de Stein y Matta refleja en sus resultados lo planteado, y demuestra como de una gran cohorte de pacientes con tromboembolia pulmonar de alto riesgo, murió solo el $15 \%$ a quienes se les realizó trombólisis, en comparación con $47 \%$ que no recibieron dicho tratamiento ${ }^{10}$.

Respecto al riesgo de sangrado asociado con el uso de trombolíticos, cabe mencionar dos aspectos puntuales. Primero, el escenario de un paciente con compromiso hemodinámico secundario a la carga embólica en la circulación pulmonar, hace evidente hacia dónde se inclinará la balanza teniendo en cuenta la relación riesgo/beneficio (de no tener contraindicaciones para dicho medicamento); segundo, la incidencia de sangrado mayor, en el peor de los escenarios, sería del $11 \%$, aunque la literatura al respecto es heterogénea, dado el uso de diferentes escalas para clasificar los sangrados en este contexto ${ }^{16}$.

\section{Caso}

Se trata de un hombre de 54 años de edad, sin antecedentes personales o familiares de importancia, quien presentaba dolor en miembro inferior derecho y edema en la pantorrilla del mismo lado desde hacía un mes. Previo al ingreso al servicio de urgencias tuvo pérdida de la conciencia y del tono postural de aproximadamente 15 minutos de duración, 


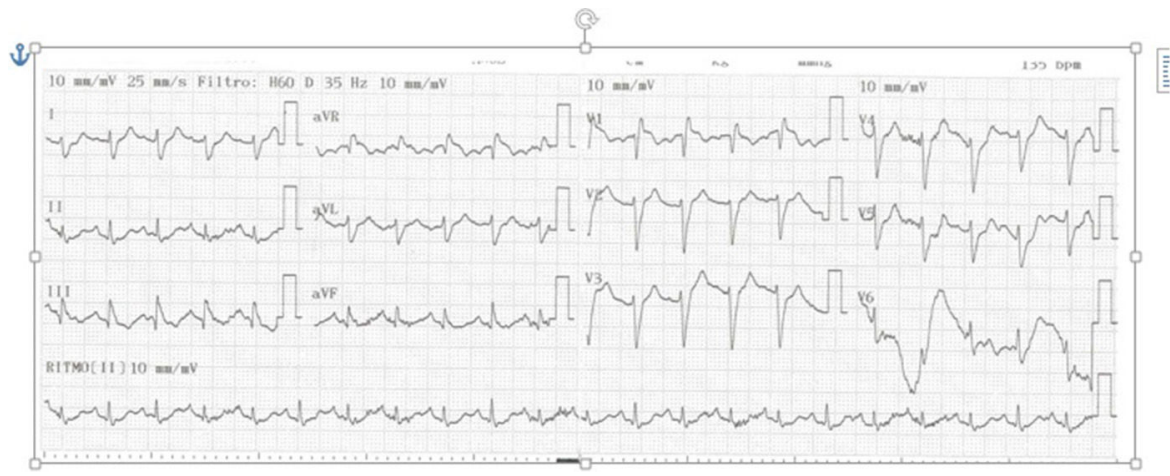

Figura 1 A la izquierda EKG de ingreso en el que se observa taquicardia sinusal, onda S profunda en DI, onda Q e inversión de onda T en DIII - S1Q3T3 (ver flechas), eje desviado a la derecha.
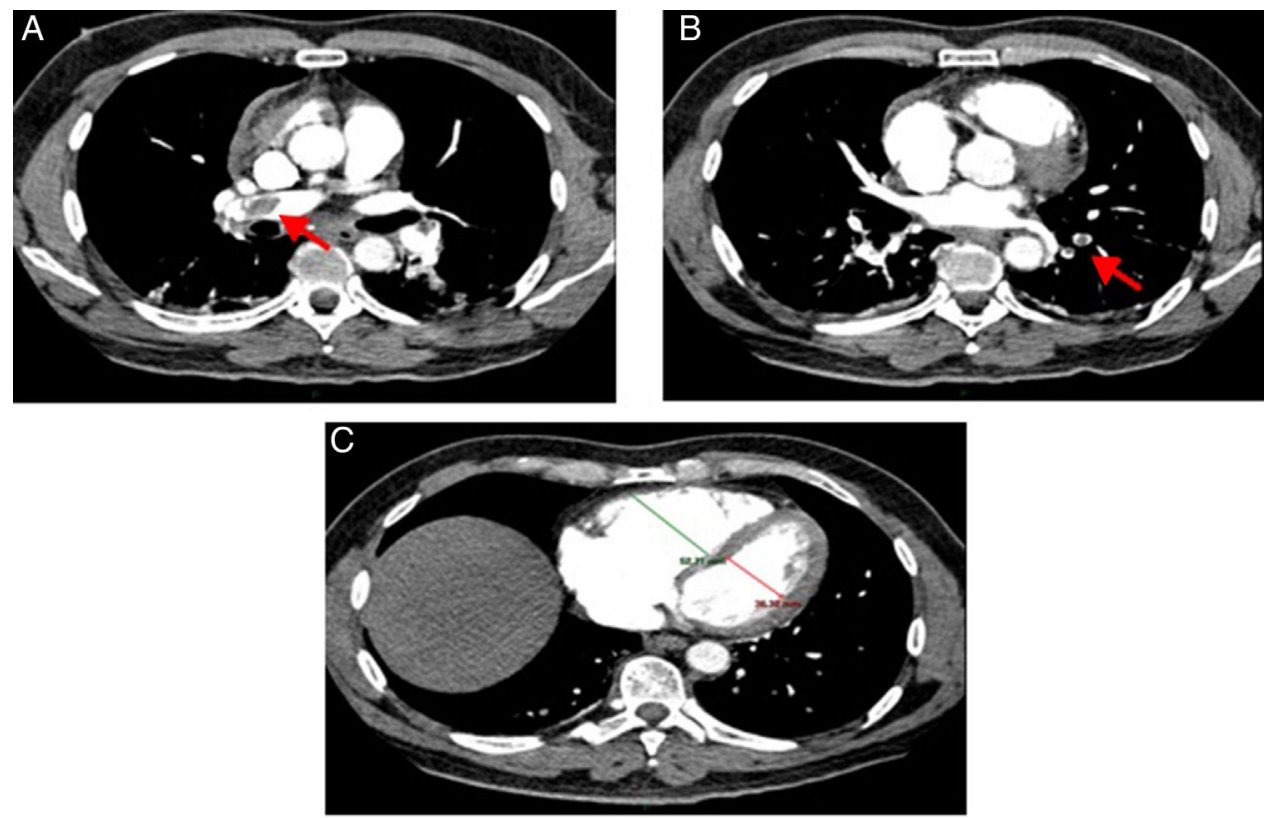

Figura 2 Tomografía contrastada de tórax con protocolo para arterias pulmonares. A) Imagen de defecto de llenado de baja atenuación en arteria pulmonar derecha y rim sign (paso de contraste alrededor del trombo). B) Defecto de llenado de baja atenuación en ramas segmentarias proximales de la arteria lobar inferior izquierda. C) aumento del diámetro del ventrículo derecho, con pérdida de la relación con el ventrículo izquierdo (relación menor a 0.9) y discreto abombamiento del septum interventricular.

con recuperación completa, sin evidencia de déficit neurológico posterior. Ingresó al servicio de urgencias alerta y refiriendo dolor torácico opresivo y muy intenso, irradiado a región dorsal, asociado a dificultad respiratoria. Al examen físico se encontró frecuencia cardiaca en 130 latidos por minuto, presión arterial en $110 / 70 \mathrm{~mm} \mathrm{Hg}$, frecuencia respiratoria 25 respiraciones por minuto y saturación de $80 \%$, a pesar de alto aporte de oxígeno; sin hallazgos patológicos durante la auscultación pulmonar. Por signos de falla respiratoria se decidió hacer intubación orotraqueal. Se ordenó electrocardiograma en el que se evidenció taquicardia sinusal, con complejo de McGinn-White o patrón S1Q3T3, con un eje desviado a la derecha (fig. 1). Seguidamente se procedió a la realización de ecocardiografía a la cabecera del paciente, observándose desplazamiento del septum interventricular hacia la izquierda, dilatación del ventrículo derecho (basal de $4,7 \mathrm{~cm}$ ), hipocinesia y función sistólica disminuida. Durante el periodo de observación presentó deterioro hemodinámico y arresto circulatorio en ritmo de actividad eléctrica sin pulso. Se realizaron maniobras de reanimación cerebrocardiopulmonar, y considerando los hallazgos clínicos, electrocardiográficos y ecocardiográficos, por la alta sospecha de tromboembolia pulmonar (clasificada como de alto riesgo) se decidió administrar trombólisis sistémica con activador tisular de plasminógeno recombinante (100 mg IV en bolo). Luego de 5 minutos de reanimación el paciente retornó a circulación espontánea en ritmo sinusal con cifras tensionales aceptables. Se decidió trasladar a angiotomografía de vasos pulmonares en la que se documentó tromboembolia pulmonar aguda bilateral (fig. 2)

Fue trasladado a la unidad de cuidados intensivos para cuidados post-reanimación; 8 horas después de la trombólisis y por mejoría clínica se decidió liberar de la ventilación mecánica y retirar tubo orotraqueal; 24 horas más tarde se llevó al servicio de hospitalización donde continuó manejo con anticoagulación parenteral (enoxaparina $1 \mathrm{mg} / \mathrm{kg}$ cada 


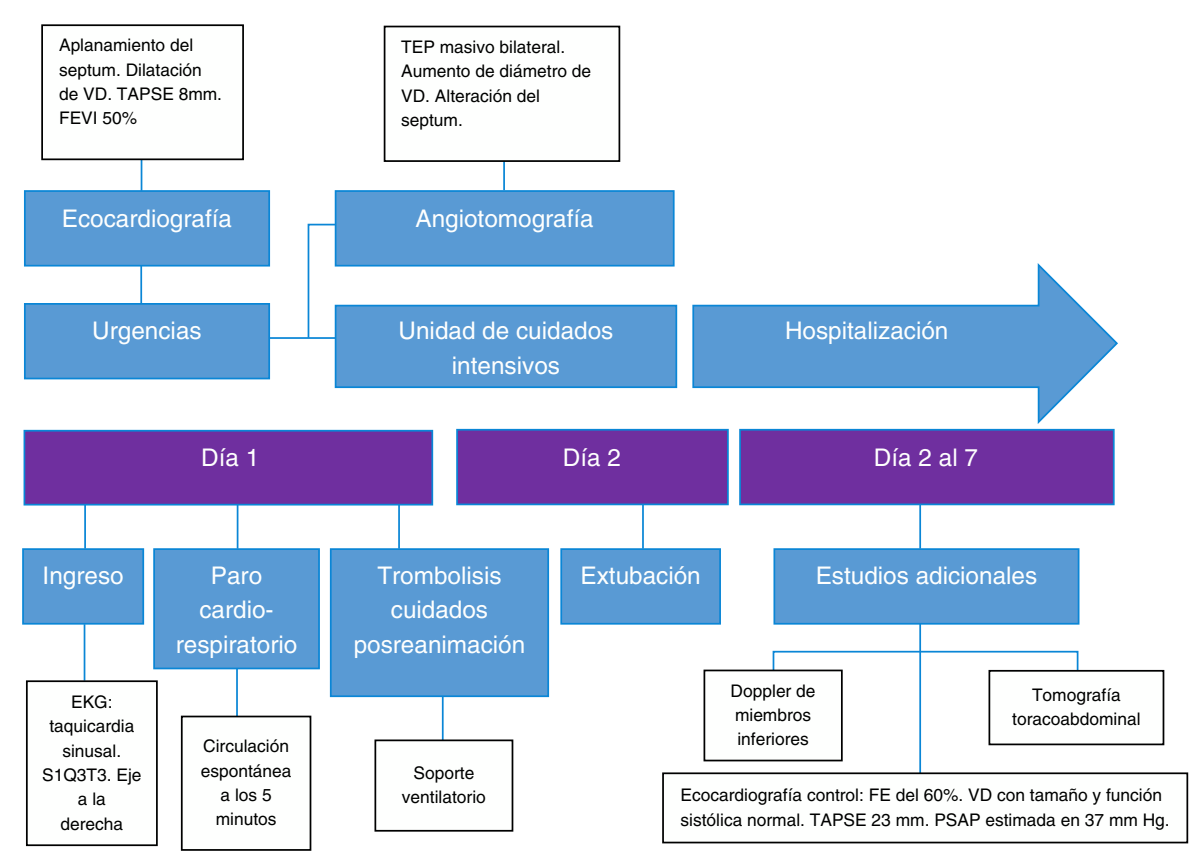

Figura 3 Diagrama que resume los principales eventos del caso clínico.

12 horas) y warfarina $5 \mathrm{mg}$ día. Se realizó adicionalmente Doppler de miembros inferiores encontrándose trombosis venosa profunda subaguda femoral distal derecha, sin recanalización en el segmento femoral y parcialmente recanalizada en vasos poplíteos e infrapoplíteos. Por ausencia de factores de riesgo para enfermedad tromboembólica venosa se inició búsqueda de neoplasia con tomografía toracoabdominal, la cual fue normal. Por otro lado, se practicó ecocardiografía de control en la que se observó fracción de expulsión del $60 \%$, ventrículo derecho con tamaño y función sistólica normal, TAPSE $23 \mathrm{~mm}$, estructuras septales íntegras y presión sistólica de la arteria pulmonar estimada en $37 \mathrm{~mm}$ $\mathrm{Hg}$.

Después de 7 días se dio el alta hospitalaria sin secuelas neurológicas y sin oxígeno suplementario. Egresó con anticoagulación oral. La figura 3 resume los eventos principales sucedidos en el paciente.

\section{Discusión}

El caso descrito es muy ilustrativo; la presentación clínica es típica: el paciente ingresó por cuadro sincopal presenciado, signos de falla respiratoria y hallazgos electrocardiográficos clásicos. Luego evolucionó con deterioro hemodinámico que lo condujo a paro cardiorrespiratorio, con actividad eléctrica sin pulso como ritmo. Ante la sospecha clínica se decidió tomar ecocardiografía a la cabecera del paciente en la cual se evidenció compromiso ventricular derecho agudo, elementos que fueron claves para el enfoque y manejo inicial. Para este caso puntual está presente una tríada (edad menor de 65 años, colapso presenciado y actividad eléctrica sin pulso como ritmo de paro), descrita por Courtney y Kline en 2005, en un esfuerzo por desarrollar una regla de decisión clínica, encontrando en su estudio que $57 \%$ de los pacientes que cumplían las tres características anotadas tenían tromboembolia pulmonar como causa del arresto cardiaco ${ }^{20}$.

Como se describió previamente, hasta el 13\% de los arrestos cardiacos de causa no clara pueden ser explicados por tromboembolia pulmonar, dato que toma mayor relevancia al ponerlo en el contexto de la enfermedad tromboembólica como la tercera causa de muerte de origen cardiovascular, lo cual indica que quizá la tromboembolia pulmonar es una causa de muerte mucho más frecuente de lo que muestran los reportes hasta el momento. Con base en el estudio realizado por Prandoni et al., la incidencia de tromboembolia pulmonar en pacientes con sintomatología cardiovascular (no necesariamente con compromiso hemodinámico), fue del $42,2 \%$ en aquellos que tenían al ingreso un dímero $D$ anormal y una probabilidad pretest baja para tromboembolia pulmonar, incluso se documentó esta entidad en el $12,7 \%$ de pacientes que tenían otra causa que explicara el síncope y en el $25,4 \%$ de quienes no tenían causa clara para éste ${ }^{8}$.

Realizar ensayos clínicos controlados para definir el impacto de la terapia trombolítica en pacientes con arresto cardiaco secundario a tromboembolia pulmonar es complejo por razones obvias. Sin embargo, al enfrentarse a un paciente con arresto cardiaco en quien se tiene sospecha o diagnóstico de tromboembolia pulmonar, hay suficientes herramientas para pensar en la terapia trombolítica temprana como la mejor opción para lograr hacer que retorne a circulación espontánea, razonamiento que se tuvo en cuenta durante el manejo del caso expuesto considerando que era la decisión más indicada. Cabe resaltar que el evento embólico pulmonar es el resultado de daño endotelial, estasis venosa, estados de hipercoagulabilidad, o la suma de ellos, y que adicionalmente la presencia del trombo en las arterias pulmonares, lleva a dilatación y disfunción ventricular derecha, daño miocárdico, disminución del volumen de fin de diástole del ventrículo izquierdo, disminución de la precarga y finalmente del gasto cardiaco, lo que desemboca en 
la causa de muerte de los pacientes con tromboembolia pulmonar: shock obstructivo ${ }^{2}$. Estos cambios fisiopatológicos se evidenciaron en la evolución clínica del caso expuesto.

Se ha propuesto que la circulación pulmonar es especialmente sensible a la terapia trombolítica dada su ventaja anatómica, que hace que el medicamento llegue allí procedente de la circulación venosa, pasando solo por las cavidades derechas del corazón. Esta hipótesis fue tomada como punto de partida en el estudio MOPETT ${ }^{13}$, en el cual se distribuyeron de manera aleatoria 121 pacientes con tromboembolia pulmonar de riesgo intermedio a utilizar "dosis seguras" de alteplase, definidas como $50 \mathrm{mg}$ para pacientes que pesaran más de $50 \mathrm{~kg}$ y $0,5 \mathrm{mg} / \mathrm{kg}$ para los que pesaran menos de $50 \mathrm{~kg}$ (61 pacientes) versus terapia anticoagulante (60 pacientes). En este estudio los desenlaces primarios fueron hipertensión pulmonar y un desenlace compuesto de hipertensión pulmonar y recurrencia de tromboembolia pulmonar a 28 meses; para ambos desenlaces la terapia fibrinolítica fue superior $\left(p<0,001\right.$ en ambos casos) ${ }^{11}$.

En 1995 Jerjes-Sanchez et al. publicaron un ensayo de distribución aleatoria, en el cual tomaron pacientes previamente sanos con alta probabilidad de tromboembolia pulmonar y los asignaron a dos grupos: trombólisis (1.500.000 $U$ de estreptoquinasa y anticoagulación con heparina) versus heparina sola. Se alcanzaron a reclutar 4 pacientes en cada grupo, tras lo cual el estudio fue suspendido por el comité de ética dados los resultados parciales: los 4 pacientes del grupo de fibrinólisis adicional a heparina estaban vivos y los 4 pacientes del grupo de heparina sola habían muerto; la diferencia fue significativa $(p<0,02)^{12}$.

Para el año 2001, Bötinger et al. publicaron un estudio prospectivo observacional, en el cual se propuso evaluar la eficacia de la terapia trombolítica (alteplase $50 \mathrm{mg}$ combinado con 5.000 unidades de heparina) en pacientes con arresto cardiaco extrahospitalario que no respondieran inicialmente a maniobras de reanimación cardiopulmonar (15 minutos de reanimación). Se incluyeron en total 90 pacientes en el estudio: 40 a quienes se les realizó el protocolo de trombólisis y 50 de una cohorte histórica que no recibió dicho tratamiento. De los pacientes a los que se les realizó trombólisis, el $68 \%$ (44\% en el control) retornó a circulación espontánea y $58 \%$ (30\% en el control) ingresó a la UCI, ambas diferencias estadísticamente significativas $(p=0,026$ y $p=0,009$, respectivamente $)^{13}$.

En 2004 se publicó el estudio TICA, un ensayo controlado en el cual se comparó la terapia trombolítica (tenecteplase $50 \mathrm{mg}$ ) versus placebo, en pacientes con arresto cardiaco extrahospitalario. Dicho estudio tenía como hipótesis que la terapia fibrinolítica temprana podría impactar favorablemente en las tasas de retorno a circulación espontánea (como novedad, se utilizó el trombolítico tan rápido como fuera posible). Se incluyeron en total 35 pacientes (19 en el grupo de intervención y 16 en el control). Pese a que finalizó de manera prematura por problemas financieros, con los pacientes reclutados se demostró una diferencia significativa en el desenlace primario (retorno a circulación espontánea): $42 \%$ en el grupo de fibrinólisis versus $6 \%$ en el grupo control ${ }^{14}$.

Para 2008, Bötinger publicó el TROICA, estudio al que ingresaron pacientes con arresto cardiaco extrahospitalario presenciado de presunto origen cardiaco. Los pacientes (1.050 en total, 525 para cada grupo) se distribuyeron en dos grupos: de intervención (tenecteplase según peso) y control al que se le administraba placebo. El desenlace primario fue sobrevida a 30 días, mientras que los secundarios fueron sobrevida al ingreso hospitalario, retorno a circulación espontánea, sobrevida a 24 horas y al alta y secuelas neurológicas. No hubo diferencias en el desenlace primario ni en los secundarios. Sin embargo, al analizar por subgrupos este estudio (la figura 2 el texto original), se encontró que de los pacientes en quienes finalmente se documentó tromboembolia pulmonar, sobrevivió un 13,3\% en el grupo de fibrinólisis versus ninguno en el grupo control ${ }^{15}$.

Con base en esta recopilación de datos en la que se evidencia que el paciente con tromboembolia pulmonar y arresto cardiaco puede beneficiarse de terapia trombolítica, y pese a que la literatura no establece claramente indicaciones, dosis y tiempo de administración de dicha terapia en el contexto de paro cardiorrespiratorio, se pudo observar que la decisión de trombólisis temprana impactó de manera favorable la mortalidad del paciente, quien fue dado de alta sin secuelas neurológicas o de otro tipo y recuperó la función cardiaca.

Finalmente, se resalta que las guías y consensos de las sociedades más reconocidas en el manejo de este tipo de pacientes, no mencionan en sus recomendaciones a aquellos que entran en arresto cardiaco por tromboembolia pulmonar o alta sospecha de la misma ${ }^{17-19}$, lo cual implica para el tratante la toma de decisiones basada en una revisión juiciosa del tema, agudeza clínica, claridad mental respecto a lo que ocurre con el paciente desde el punto de vista fisiopatológico e instauración temprana de reperfusión.

\section{Conclusión}

De acuerdo con lo planteado en el desarrollo de este reporte, la tromboembolia pulmonar no es una entidad tan inusual como habitualmente se cree. En los casos en los que esta lleva a arresto cardiaco, la supervivencia está íntimamente ligada con el momento en el cual se inicie la terapia de reperfusión, de ahí la relevancia del alto índice de sospecha y proceder de manera lógica y rápida.

Respecto al caso expuesto, se puede concluir que después de un enfoque correcto y de la valoración adecuada de los hallazgos clínicos y las ayudas diagnósticas, la trombólisis fue un procedimiento acertado según la evidencia previamente descrita, que impactó positivamente la supervivencia y la función cardiopulmonar a corto y largo plazo luego de retirar el estrés mecánico del ventrículo derecho de una manera rápida y eficaz.

\section{Responsabilidades éticas}

Protección de personas y animales. Los autores declaran que para esta investigación no se han realizado experimentos en seres humanos ni en animales.

Confidencialidad de los datos. Los autores declaran que en este artículo no aparecen datos de pacientes.

Derecho a la privacidad y consentimiento informado. Los autores declaran que en este artículo no aparecen datos de pacientes. 


\section{Conflicto de intereses}

\section{Ninguno.}

\section{Bibliografía}

1. Takach Lapner S, Kearon C. Diagnosis and management of pulmonary embolism. BMJ. 2013;346:f757.

2. Wood KE. Major pulmonary embolism: review of a pathophysiologic approach to the golden hour of hemodynamically significant pulmonary embolism. Chest. 2002;121:877-905.

3. Cohen AT, Argnelli G, Anderson FA, Arcelus JI, Bergqvist D, Brecht JG, et al. Venous thromboembolism (VTE) in Europe The number of VTE events and associated morbidity and mortality. Thromb Haemost. 2007;98:756-64.

4. Logan JK, Pantle H, Huiras P, Bessman E, Bright L. Evidencebased diagnosis and thrombolytic treatment of cardiac arrest or periarrestal due to suspected pulmonary embolism. Am J Emerg Med. 2014;32:789-96.

5. Goldhaber SZ, Visani L, De Rosa M. Acute pulmonary embolism: clinical outcomes in the International Cooperative Pulmonary Embolism Registry (ICOPER). Lancet. 1999;353:1389-96.

6. Laporte S, Mismetti P, Décousus H, Uresandi F, Otero R, Lobo JL, et al. Clinical predictors for fatal pulmonary embolism in 15520 patients with venous thromboembolism Findings from Registro Informatizado de la Enfermedad TromboEmbolica venosa (RIETE) Registry. Circulation. 2008;117:6-1711.

7. Stein PD, Beemath A, Matta F, Weg JG, Yusen RD, Hales CA, et al. Clinical characteristics of patients with acute pulmonary embolism: data from PIOPED II. Am J Med. 2007;120:9-871.

8. Prandoni $P$, Lensing AWA, Prins $M H$, Ciammaichella $M$, Perlati $M$, Numoli $N$, et al. Prevalence of pulmonary embolism among patients hospitalized for syncope. N Eng J Med. 2016;375:31-1524

9. Schouten HJ, Geersing GJ, Oudega R, van Delden JJ, Moons KG, Koek HL. Accuracy of the Wells clinical prediction rule for pulmonary embolism in older ambulatory adults. J Am Geriatr Soc. 2014;62:41-2136.

10. Stein PD, Matta F. Thrombolytic therapy in unstable patients with acute pulmonary embolism: Saves lives but underused. Am J Med. 2012;125:70-465.
11. Sharifi M, Bay C, Skrocki L, Rahimi F, Mehdipour M. Moderate pulmonary embolism treated with thrombolysis. Am J Cardiol. 2013;111:7-273.

12. Jerjes-Sanchez J, Ramirez-Rivera A, de Lourdes García M, Arriaga-Nava R, Valencia S, Rosado-Buzzo A, et al. Streptokinase and heparin versus heparin alone in massive pulmonary embolism: a randomized controled trial. J Thromb Thrombolysis. 1995;2:9-227.

13. Bötinger BW, Bode C, Kern S, Gries A, Gust A, Glätzer R, et al. Efficacy and safety of thrombolytic therapy after initially unsuccesful cardiopulmonary resuscitation: a prospective clinical trial. Lancet. 2001;357:5-1583.

14. Fatovich DM, Dobb GJ, Clugston RA. A pilot randomised trial of thrombolysis in cardiac arrest (the TICA trial). Resuscitation. 2004;61:13-309.

15. Bötinger BW, Arnzt HR, Chamberlain DA, Bluhmki E, Belmans A, Danays T, et al. Thrombolysis during resuscitation for out-of-hospital cardiac arrest. N Eng J Med. 2008;359: 62-2651.

16. Konstantinides SV, Barco S. Prevention of early complications and late consequences after acute pulmonary embolism: focus on reperfusion techniques. Thromb Res. 2017, pii:S00493848(17)30352-3.

17. Konstantinides SV, Torbicki A, Agnelli G, Danchin N, Fitzmaurice D, Galie N, et al. 2014 ESC guidelines on the diagnosis and management of acute pulmonary embolism: the task force for the diagnosis and management of acute pulmonary embolism of the European Society of Cardiology (ESC) Endorsed by the European Respiratory Society (ERS). Eur Heart J. 2014;35: 73-3033.

18. Jaff MR, McMurtry MS, Archer SL, Cushman M, Goldenberg N, Goldhaber SZ, et al. Management of massive and submassive pulmonary embolism, iliofemoral deep vein thrombosis, and chronic thromboembolic pulmonary hypertension: a scientific statement from the American Heart Association. Circulation. 2011;123:830-1788.

19. Kearon C, Akl EA, Ornelas J, Blaivas A, Jimenez D, Bounameaux $\mathrm{H}$, et al. Antithrombotic therapy for VTE disease: CHEST guideline and expert panel report. Chest. 2016;149:52-315.

20. Courtney DM, Kline JA. Prospective use of a clinical decision rule to identify pulmonary embolism as likely cause of outpatient cardiac arrest. Resuscitation. 2005;65:57-64. 